\title{
Atividade Física, Pressão Arterial e Variabilidade da Frequência Cardíaca em Crianças e Adolescentes
}

\section{Physical Activity, Blood Pressure and Heart Rate Variability in Children and Adolescents}

\author{
Juliano Casonatto*a; Elisângela Miyazawa ${ }^{a}$; Daniele Zago ; Diego Christofaro ${ }^{\text {b; }}$ Kamila Grandolfi;
}

${ }^{a}$ UNOPAR, Programa de Pós-Graduação Stricto Sensu em Exercício Físico na Promoção da Saúde. PR, Brasil.

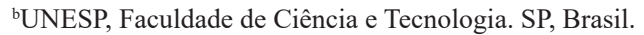

*E-mail: juliano2608@hotmail.com

\begin{abstract}
Resumo
A atuação do sistema nervoso autonômico está diretamente associada à condição fisiológica geral, sendo utilizada inclusive como demarcador de condição clínica, bem como indicador de risco para doenças cardiovasculares. No entanto, os estudos dessas relações têm sido conduzidos prioritariamente em população adulta. Nesse sentido, o objetivo do estudo foi analisar a associação entre prática habitual de atividade física, pressão arterial (PA) e variabilidade da frequência cardíaca (VFC) em crianças e adolescentes em Londrina, Paraná, Brasil. Estudo apresenta delineamento transversal, de modo que a amostra foi composta por 42 alunos da rede estadual de ensino, de ambos os sexos com idade entre 10 e 17 anos. Avaliou-se a massa corporal e estatura. Foram coletados ainda valores de PA sistólica e diastólica, VFC e a prática habitual de atividades físicas (PHAF) em diferentes domínios (geral, tempo livre, lazer e escola). O teste qui-quadrado foi empregado para avaliar as associações. Não foram identificadas associações estatisticamente significativas entre as variáveis cardiovasculares e a PHAF. A prática geral de atividades físicas habituais, bem como seus diferentes domínios, sejam eles no lazer, escola e tempo livre, não se associaram à valores aumentados de pressão arterial, bem como com os indicadores do comportamento autonômico de crianças e adolescentes.
\end{abstract}

Palavras-chave: Atividade Física. Pressão Arterial. Sistema Neural. Criança e Adolescente.

\begin{abstract}
The role of the autonomic nervous system is directly related to the general physiological condition, including being used as a clinical condition path as well as a risk indicator for cardiovascular diseases. However, studies of these relationships have been conducted primarily in the adult population. Thus, the study aimed to analyze the association among habitual practice of physical activity, blood pressure (BP) and heart rate variability (HRV) in children and adolescents from Londrina, Paraná, Brazil. The study presents a cross-sectional design so that the sample consisted of 42 students from the state school system, of both sexes, aged between 10 and 17 years. Body mass and height were evaluated. Systolic and diastolic BP values, HRV, and habitual physical activity (HPA) were also collected in different domains (general, free time, leisure, and school). The chi-square test was used to assess associations. No statistically significant associations were identified between cardiovascular variables and HPA. The general practice of habitual physical activities, as well as their different domains, either leisure, school, or free time, were not associated with increased blood pressure values, as well as with the indicators of the autonomic nervous system in children and adolescents.
\end{abstract}

Keywords: Physical Activity. Blood Pressure. Neural System. Children and Adolescents.

\section{Introdução}

A variabilidade da frequência cardíaca (VFC) reflete as alterações batimento a batimento nos intervalos R-R, que estão relacionadas à interação contínua entre os ramos parassimpáticos e simpáticos do sistema nervoso autônomo (Task Force of the European Society of Cardiology and the North American Society of Pacing and Electrophysiology, 1996). A análise da VFC fornece um método não invasivo e simples para avaliar a função autonômica. Como tal, tem sido empregado nas mais diversas áreas da pesquisa clínica (LEES et al., 2018). Medidas da VFC também podem oferecer biomarcadores elétricos promissores com potencial de prever a mortalidade cardíaca e dessa forma, apropriadas para aplicação prognóstica (SEN; MCGILL, 2018).

A hipertensão, por sua vez, é uma condição crônica que pode levar a doenças cardíacas, acidente vascular cerebral, dentre outras doenças que podem resultar em morte prematura (KUNG; XU, 2015), além do que, valores elevados de VFC estão associados a uma menor taxa de morbimortalidade (GOIT; ANSARI, 2016). Além dessa relação entre hipertensão e VFC, um corpo crescente de literatura tem demonstrado consistentemente que a VFC pode ser um bom indicador de saúde geral, seja ela física ou emocional (JOYCE; BARRETT, 2019). O padrão da VFC é complexo, porém, no coração saudável, a VFC pode ser decomposta em um pequeno conjunto de oscilações sobrepostas. A complexidade está organizada no sentido de que a mesma pode ser descrita usando um conjunto de fórmulas, que denotam o padrão de regulação da frequência cardíaca pelo sistema nervoso autonômico. Esse sistema é o principal coordenador dessas oscilações, que refletem um conjunto de reflexos neurais que ajudam a controlar várias outras funções do corpo (LEHRER 
et al., 2020).

A atividade física pode aumentar a VFC total em idosos (STEIN et al., 1999), atletas (PLEWS et al., 2013) e jovens (HEFFERNAN et al., 2007). Estudos observacionais (SOARES-MIRANDA et al., 2014) e de intervenção (KIVINIEMI et al., 2014) demonstraram que a modulação vagal cardíaca é significativamente mais alta em indivíduos que praticam mais atividade física, em comparação com seus pares mais sedentários. Portanto, a prática de atividade física tem sido proposta como uma intervenção preventiva para limitar ou mesmo reverter o declínio na VFC.

No entanto, a investigação dos fatores relacionados ao aumento da pressão arterial (PA) em crianças e adolescentes é significativamente mais limitada e constitui uma tarefa de alta complexidade, uma vez que envolve o controle de variáveis de natureza comportamental, como por exemplo, os diferentes domínios da prática habitual de atividades físicas (PHAF), que podem ser significativamente diferentes entre distintos grupos populacionais.

Nesse sentido, a obtenção de informações acerca dos fatores de risco associados ao desenvolvimento de doenças de caráter crônico-degenerativo em crianças e adolescentes poderá contribuir para a proposição de ações mais eficientes voltadas a prevenção desses agravos a saúde. Desse modo, o objetivo do presente estudo foi investigar a associação entre PHAF, PA e VFC em crianças e adolescentes.

\section{Material e Métodos}

\subsection{Participantes}

A amostra foi composta por 42 participantes de ambos os sexos, com idades entre 10 e 17 anos matriculados no ensino fundamental e médio na rede estadual de ensino da cidade de Londrina, Paraná, Brasil. Para inclusão no estudo, os escolares deveriam se apresentar saudáveis, ou seja, não possuir diagnóstico formal de doenças crônicas, bem como limitações osteomiarticulares que comprometessem a prática de atividades físicas. Foram excluídos do estudo aqueles participantes que apresentavam transitoriamente alguma lesão que pudesse interferir na prática habitual de atividades físicas reportada. Nenhum participante reportou a ingestão de medicamentos de so contínuo. Os responsáveis assinaram um termo de consentimento livre e esclarecido sobre os procedimentos em que os sujeitos foram submetidos. Esse estudo foi submetido e aprovado por Comitê de Ética em Pesquisa Institucional (Parecer № 295/07 - CAAE No 028.0.268.000-07).

\subsection{Desenho do estudo}

O presente estudo se caracteriza pelo delineamento transversal e foi realizado em dois estabelecimentos de ensino da rede pública estadual, selecionados por conveniência.
Inicialmente foi solicitada autorização nos colégios para que a pesquisa pudesse ser realizada. Após esse momento, a pesquisa foi devidamente esclarecida aos alunos e o termo de consentimento livre e esclarecido foi entregue. Puderam participar das avaliações os alunos que apresentaram termo de consentimento livre e esclarecido assinado por seus pais ou responsáveis.

As avaliações foram iniciadas pelo questionário de PHAF. Na sequência, os sujeitos foram encaminhados para a avaliação das medidas antropométricas (estatura e massa corporal). Em seguida cada avaliado recebeu uma explicação, e se fosse necessário, ajuda para posicionar o monitor de frequência cardíaca para que pudesse ser iniciada avaliação da VFC. Nesta avaliação, os indivíduos permaneceram em posição supina, impossibilitados de falar e se movimentar por um período de 10 minutos. Quando finalizada essa etapa os avaliados foram orientados para que realizassem movimentos lentos até chegar à posição sentada, para que fosse realizada a medida da PA.

\subsection{Antropometria}

Para as avaliações antropométricas, todos os adolescentes estavam vestidos com roupas leves e descalços. A massa corporal foi avaliada por meio de uma balança eletrônica com capacidade máxima de $150 \mathrm{~kg}$. A estatura foi aferida por meio de um estadiômetro portátil. Todos as avaliações foram realizadas seguindo padronização previamente estabelecida na literatura (GORDON et al., 1988).

\subsection{Prática habitual de atividades físicas (PHAF)}

A PHAF foi avaliada por meio de um questionário utilizando um inquérito especificamente desenvolvido para essa finalidade (BAECKE et al., 1982) validado para aplicação em populações pediátricas brasileiras (GUEDES; LOPES; et al., 2006). O mesmo foi respondido pelos próprios indivíduos, sendo que os pesquisadores ficaram a disposição para estritamente solucionar dúvidas relativas à interpretação das questões, com especial atenção àqueles com idade inferior à 14 anos, conforme recomendações sugeridas para aplicação desse instrumento (GUEDES; LOPES; et al., 2006). Esse instrumento fornece um escore adimensional considerando os domínios de PHAF no ambiente escolar, lazer, tempo livre e a prática de atividade física total (escola + lazer + atividade esportiva fora do ambiente escolar). Aplicou-se uma análise normativa de modo que foi considerada "alta" PHAF aqueles participantes situados no quarto quartil, e "baixa" PHAF os inseridos nos demais quartis (primeiro, segundo e terceiro) (variável categórica). Omesmo procedimento de dicotomização foi aplicado para os diferentes domínios da PHAF.

\subsection{Pressão arterial (PA)}

Para a avaliação da PA foi utilizado um aparelho 
oscilométrico automático (Omron - HEM 742) previamente validado para adolescentes (CHRISTOFARO et al., 2009). Os sujeitos foram mantidos em repouso na posição sentada por 10 minutos antes da avaliação da PA. Os procedimentos para medida foram aqueles previamente estabelecidos na literatura (PICKERING et al., 2005). Os critérios adotados para a classificação dos sujeitos como normotensos ou como portadores de PA elevada foram aqueles estabelecidos pelo Clinical Practice Guideline for Screening and Management of High Blood Pressure in Children and Adolescents (FLYNN et al., 2017).

\subsection{Variabilidade da frequência cardíaca}

A VFC foi monitorada em repouso por meio de um monitor cardíaco (Polar RS800CX, Kempele, Finlândia), previamente validado (QUINTANA et al., 2012). Os participantes permaneceram deitados em um ambiente calmo, silencioso e termoneutro $\left(22^{\circ} \mathrm{C}\right.$ a $\left.24^{\circ} \mathrm{C}\right)$ durante o monitoramento. Os intervalos $\mathrm{R}-\mathrm{R}$ gravados foram transferidos para um computador usando um software específico (Polar ProTrainer, Kempele, Finlândia). A transformada rápida de Fourier foi aplicada para quantificar as baixas (LF) e altas (HF) frequências em unidades normalizadas, de acordo com a Força-Tarefa da Sociedade Europeia de Cardiologia e a Sociedade Norte-Americana de Estimulação e Eletrofisiologia (Heart rate variability: standards of measurement, physiological interpretation and clinical use. Task Force of the European Society of Cardiology and the North American Society of Pacing and Electrophysiology, 1996).

A análise no domínio do tempo foi obtida por meio do índice RMSSD (raiz quadrada da média da soma dos quadrados das diferenças entre os intervalos RR adjacentes). A análise do intervalo de variação foi de 10 minutos, usando o software Kubios HRV (versão 2.2 - Kuopio, Finlândia).

A dicotomização das variáveis foi estabelecida por critério normativo, de modo que aqueles sujeitos pertencentes ao primeiro quartil foram classificados como portadores de "baixa" e aqueles classificados nos demais quartis como portadores de "alta" VFC de acordo com os indicadores analisados (RMSSD, LF, HF e razão LF/HF).

\subsection{Tratamento estatístico}

A distribuição dos dados foi verificada por meio do teste "Shapiro Wilk". Os procedimentos de estatística descritiva foram aplicados para caracterização da amostra (média e desvio padrão). O teste "T" de Student para amostras independentes foi empregado para comparações das características gerais da amostra entre os sexos. Para identificação das possíveis associações entre os domínios da PHAF e as variáveis cardiovasculares foi utilizado o teste qui-quadrado. O nível de significância estabelecido foi de $P<0,05$. Todas as análises foram realizadas no software SPSS 17.0.

\section{Resultados e Discussão}

As características gerais e as variáveis cardiovasculares da amostra são apresentadas no Quadro 1. Não foram identificadas diferenças estatisticamente significativas entre os participantes alocados nos grupos de "baixa" ou "alta" PHAF nos componentes antropométricos e cardiovasculares.

Quadro 1 - Características gerais da amostra em valores médios e o teste " $T$ " de Student para amostras independentes

\begin{tabular}{|l|c|c|c|}
\hline \multirow{2}{*}{ Variáveis } & $\begin{array}{c}\text { Baixa PHAF } \\
(\mathbf{n = 3 2})\end{array}$ & $\begin{array}{c}\text { Alta PHAF } \\
(\mathbf{n = 1 0 )}\end{array}$ & \multirow{2}{*}{$\boldsymbol{P}$} \\
\cline { 2 - 3 } & Média (DP) & Média (DP) & \\
\hline Idade (anos) & $13,0(2,0)$ & $12,5(1,3)$ & 0,456 \\
\hline MC (kg) & $48(13)$ & $44(10)$ & 0,346 \\
\hline Estatura (m) & $1,56(0,12)$ & $1,56(0,08)$ & 0,989 \\
\hline PAS (mmHg) & $110(9,9)$ & $107(6,3)$ & 0,383 \\
\hline PAD (mmHg) & $70(5,6)$ & $67(3,8)$ & 0,201 \\
\hline RMSSD (ms) & $245(190)$ & $198(126)$ & 0,477 \\
\hline LF (n.u.) & $43(17)$ & $42(17)$ & 0,917 \\
\hline HF (n.u.) & $28(14)$ & $24(8)$ & 0,376 \\
\hline LF/HF (\%) & $2,47(3,00)$ & $2,30(2,06)$ & 0,866 \\
\hline PHAF=prican
\end{tabular}
$\mathrm{PHAF}=$ prática habitual de atividades físicas; $\mathrm{DP}=$ desvio padrão; $\mathrm{MC}=$ Massa corporal; $\mathrm{PAS}=$ pressão arterial sistólica; $\mathrm{PAD}=$ pressão arterial diastólica; $\mathrm{RMSSD}=$ raiz quadrada da média do quadrado das diferenças entre intervalos $\mathrm{RR}$ normais adjacentes; $\mathrm{LF}=$ componente de baixa frequência; $\mathrm{HF}=$ componente de alta frequência; $\mathrm{LF} / \mathrm{HF}=$ razão entre componente de baixa frequência e componente de alta frequência.

Fonte: Dados da pesquisa.

Não foram identificadas associações estatisticamente significativas entre as variáveis cardiovasculares e a PHAF (Quadro 2). Em relação às variáveis relacionadas à VFC, os sujeitos com alta PHAF apresentaram valores mais elevados para RMSSD, LF e LF/HF. Por outro lado, os sujeitos classificados com alta PHAF tendem a possuir menores valores de HF.

Quadro 2 - Associação entre as variáveis cardiovasculares e a prática habitual de atividades físicas (PHAF)

\begin{tabular}{|c|c|c|c|c|c|}
\hline \multirow{2}{*}{\multicolumn{2}{|c|}{$\begin{array}{c}\text { Variáveis } \\
\text { Cardiovasculares }\end{array}$}} & $\begin{array}{c}\text { Baixa PHAF } \\
(\mathrm{n}=32)\end{array}$ & $\begin{array}{l}\text { Alta PHAF } \\
(\mathrm{n}=10)\end{array}$ & \multirow[t]{2}{*}{$\chi^{2}$} & \multirow[t]{2}{*}{$P$} \\
\hline & & $n(\%)$ & $n(\%)$ & & \\
\hline \multirow{2}{*}{$\begin{array}{c}\text { PAS } \\
(\mathrm{mmHg})\end{array}$} & \begin{tabular}{|l|} 
Normal \\
\end{tabular} & $26(74,3)$ & $9(25,7)$ & \multirow{2}{*}{0,420} & \multirow{2}{*}{0,517} \\
\hline & Elevada & $6(85,7)$ & $1(14,3)$ & & \\
\hline \multirow{2}{*}{$\begin{array}{c}\text { PAD } \\
(\mathrm{mmHg})\end{array}$} & Normal & $28(73,7)$ & $10(26,3)$ & \multirow{2}{*}{1,382} & \multirow{2}{*}{0,240} \\
\hline & \begin{tabular}{|l|} 
Elevada \\
\end{tabular} & $4(100,0)$ & $0(0,0)$ & & \\
\hline \multirow{2}{*}{$\begin{array}{l}\text { RMSSD } \\
(\mathrm{ms})\end{array}$} & Baixa & $8(80,0)$ & $2(20,0)$ & \multirow{2}{*}{0,105} & \multirow{2}{*}{0,746} \\
\hline & Elevada & $24(75,0)$ & 81 & & \\
\hline \multirow{2}{*}{ LF (n.u.) } & Baixo & $9(90,0)$ & $1(10,0)$ & \multirow{2}{*}{1,380} & \multirow{2}{*}{0,240} \\
\hline & Elevado & $23(71,9)$ & $9(28,1)$ & & \\
\hline \multirow{2}{*}{$\begin{array}{l}\text { HF } \\
\text { (n.u.) }\end{array}$} & Baixo & $7(70,0)$ & $3(30,0)$ & \multirow{2}{*}{0,277} & \multirow{2}{*}{0,598} \\
\hline & Elevado & $25(78,1)$ & $7(21,9)$ & & \\
\hline \multirow{2}{*}{$\begin{array}{c}\mathrm{LF} / \mathrm{HF} \\
(\%)\end{array}$} & Baixo & $9(90,0)$ & $1(10,0)$ & \multirow{2}{*}{1,380} & \multirow{2}{*}{0,240} \\
\hline & Elevado & $23(71,9)$ & $9(28,1)$ & & \\
\hline
\end{tabular}

PAS $=$ pressão arterial sistólica; $\mathrm{PAD}=$ pressão arterial diastólica; RMSSD $=$ raiz quadrada da média do quadrado das diferenças entre intervalos $\mathrm{RR}$ normais adjacentes; $\mathrm{LF}=$ componente de baixa frequência; $\mathrm{HF}=$ componente de alta frequência; $\mathrm{LF} / \mathrm{HF}=$ razão entre componente de baixa frequência e componente de alta frequência.

Fonte: Dados da pesquisa. 
Os dados de associação entre as variáveis cardiovasculares e a prática habitual de atividades físicas no ambiente escolar (PHAFE) são apresentados na Tabela 3. Não foram identificadas associações estatisticamente significativas entre as variáveis cardiovasculares e a PHAFE.

Daqueles sujeitos portadores de PA sistólica e diastólica elevada, a maioria apresenta baixa prática habitual de atividades físicas na escola (PHAFE). Considerando as proporções absolutas, àqueles sujeitos com baixa PHAFE também apresentaram maior prevalência de menores valores para RMSSD e LF. Em relação ao HF o comportamento foi inverso.

Quadro 3 - Associação entre as variáveis cardiovasculares e a prática habitual de atividades físicas na escola (PHAFE).

\begin{tabular}{|c|c|c|c|c|c|}
\hline \multirow{2}{*}{\multicolumn{2}{|c|}{\begin{tabular}{|c|} 
Variáveis \\
Cardiovasculares
\end{tabular}}} & $\begin{array}{c}\text { Baixa } \\
\text { PHAFE } \\
(n=26)\end{array}$ & $\begin{array}{c}\text { Alta } \\
\text { PHAFE } \\
(n=16)\end{array}$ & \multirow[t]{2}{*}{$\chi^{2}$} & \multirow[t]{2}{*}{$\boldsymbol{P}$} \\
\hline & & \multicolumn{2}{|l|}{$n(\%)$} & & \\
\hline \multirow{2}{*}{$\begin{array}{c}\text { PAS } \\
(\mathrm{mmHg})\end{array}$} & Normal & $20(57,1)$ & $15(42,9)$ & \multirow{2}{*}{2,019} & \multirow{2}{*}{0,155} \\
\hline & Elevada & $6(85,7)$ & $1(14,3)$ & & \\
\hline \multirow{2}{*}{$\begin{array}{c}\text { PAD } \\
(\mathrm{mmHg})\end{array}$} & Normal & $23(60,5)$ & $15(39,5)$ & \multirow{2}{*}{0,321} & \multirow{2}{*}{0,571} \\
\hline & Elevada & $3(75,0)$ & $1(2$ & & \\
\hline \multirow{2}{*}{$\begin{array}{c}\text { RMSSD } \\
(\mathrm{ms})\end{array}$} & Baixa & $7(70,0)$ & $3(30,0)$ & \multirow{2}{*}{0,365} & \multirow{2}{*}{0,546} \\
\hline & Elevada & $19(59,4)$ & $13(40,6)$ & & \\
\hline \multirow{2}{*}{ LF (n.u.) } & Baixo & $7(70,0)$ & $3(30,0)$ & \multirow{2}{*}{0,365} & \multirow{2}{*}{0,546} \\
\hline & Elevado & $19(59,4)$ & $13(40,6)$ & & \\
\hline \multirow{2}{*}{ HF (n.u.) } & Baixo & $5(50,0)$ & $5(50,0)$ & \multirow{2}{*}{0,789} & \multirow{2}{*}{0,374} \\
\hline & Elevado & $21(65,6)$ & $11(34,4)$ & & \\
\hline \multirow{2}{*}{$\begin{array}{c}\mathrm{LF} / \mathrm{HF} \\
(\%)\end{array}$} & Baixo & $6(60,0)$ & $4(40,0)$ & \multirow{2}{*}{0,020} & \multirow{2}{*}{0,887} \\
\hline & Elevado & $20(62,5)$ & $12(37,5)$ & & \\
\hline
\end{tabular}

$\mathrm{PAS}=$ pressão arterial sistólica; $\mathrm{PAD}=$ pressão arterial diastólica; $\mathrm{RMSSD}=$ raiz quadrada da média do quadrado das diferenças entre intervalos RR normais adjacentes; $\mathrm{LF}=$ componente de baixa frequência; $\mathrm{HF}=$ componente de alta frequência; $\mathrm{LF} / \mathrm{HF}=$ razão entre componente de baixa frequência e componente de alta frequência.

Fonte: Dados da pesquisa.

O Quadro 4 apresenta a associação entre as variáveis cardiovasculares e a prática habitual de atividades físicas no lazer (PHAFL). Quanto aos indivíduos que possuem baixa PHAFL, a maioria destes apresentou valores elevados de PAD (proporções absolutas). Além disso, a maior parte dos indivíduos com alta PHAFL demonstrou valores elevados de LF. As demais variáveis relacionadas à VFC não apresentaram variações importantes entre aqueles indivíduos com baixa e alta PHAFL. Não foram encontradas associações estatisticamente significativas entre as variáveis cardiovasculares e a PHAFL.
Quadro 4 - Associação entre as variáveis cardiovasculares e a prática habitual de atividades físicas no lazer (PHAFL)

\begin{tabular}{|c|c|c|c|c|c|}
\hline \multirow{2}{*}{\multicolumn{2}{|c|}{$\begin{array}{c}\text { Variáveis } \\
\text { Cardiovasculares }\end{array}$}} & $\begin{array}{c}\text { Baixa } \\
\text { PHAFL } \\
(n=31)\end{array}$ & $\begin{array}{c}\text { Alta } \\
\text { PHAFL } \\
(n=11)\end{array}$ & \multirow[t]{2}{*}{$\chi^{2}$} & \multirow[t]{2}{*}{$\boldsymbol{P}$} \\
\hline & & $n(\%)$ & $n(\%)$ & & \\
\hline \multirow{2}{*}{$\begin{array}{c}\text { PAS } \\
(\mathrm{mmHg})\end{array}$} & Normal & $26(74,3)$ & $9(25,7)$ & \multirow{2}{*}{0,025} & \multirow{2}{*}{0,875} \\
\hline & Elevada & $5(71,4)$ & $2(28,6)$ & & \\
\hline \multirow{2}{*}{$\begin{array}{c}\text { PAD } \\
(\mathrm{mmHg})\end{array}$} & Normal & $27(71,1)$ & $11(28,9)$ & \multirow{2}{*}{1,569} & \multirow{2}{*}{0,210} \\
\hline & Elevada & $4(100,0)$ & $0(0,0)$ & & \\
\hline \multirow{2}{*}{$\begin{array}{c}\text { RMSSD } \\
(\mathrm{ms})\end{array}$} & Baixa & $8(80,0)$ & $2(20,0)$ & \multirow{2}{*}{0,260} & \multirow{2}{*}{0,610} \\
\hline & Elevada & $23(71,9)$ & $9(28,1)$ & & \\
\hline \multirow{2}{*}{ LF (n.u.) } & Baixo & $9(90,0)$ & $1(10,0)$ & \multirow{2}{*}{1,780} & \multirow{2}{*}{0,182} \\
\hline & \begin{tabular}{|l|} 
Elevado \\
\end{tabular} & $22(68,8)$ & $10(31,3)$ & & \\
\hline \multirow{2}{*}{ HF (n.u.) } & Baixo & $7(70,0)$ & $3(30,0)$ & \multirow{2}{*}{0,099} & \multirow{2}{*}{0,754} \\
\hline & Elevado & $24(75,0)$ & $8(25,0)$ & & \\
\hline \multirow{2}{*}{$\begin{array}{c}\mathrm{LF} / \mathrm{HF} \\
(\%)\end{array}$} & Baixo & $8(80,0)$ & $2(20,0)$ & \multirow{2}{*}{0,260} & \multirow{2}{*}{0,610} \\
\hline & Elevado & $23(71,9)$ & $9(28,1)$ & & \\
\hline
\end{tabular}

$\mathrm{PAS}=$ pressão arterial sistólica; $\mathrm{PAD}=$ pressão arterial diastólica; RMSSD = raiz quadrada da média do quadrado das diferenças entre intervalos RR normais adjacentes; $\mathrm{LF}=$ componente de baixa frequência; $\mathrm{HF}=$ componente de alta frequência; $\mathrm{LF} / \mathrm{HF}=$ razão entre componente de baixa frequência e componente de alta frequência.

Fonte: Dados da pesquisa.

O Quadro 5 apresenta a associação entres as variáveis cardiovasculares e prática habitual de atividade física no tempo livre (PHAFTL). Os valores de pressão arterial sistólica (PAS) associados à PHAFTL foram controversos aos valores de PAS associados aos demais domínios de PHAF. Quando associados à baixa PHAFTL a maioria dos indivíduos apresentou valores normais de PAD. Não foram encontradas associações estatisticamente significativas entre as variáveis cardiovasculares e a PHAFTL.

Quadro 5 - Associação entre as variáveis cardiovasculares e a prática habitual de atividades físicas no tempo livre (PHAFTL)

\begin{tabular}{|c|c|c|c|c|c|}
\hline \multirow{2}{*}{\multicolumn{2}{|c|}{$\begin{array}{c}\text { Variáveis } \\
\text { Cardiovasculares }\end{array}$}} & $\begin{array}{c}\text { Baixa } \\
\text { PHAFTL } \\
(n=31)\end{array}$ & $\begin{array}{c}\text { Alta } \\
\text { PHAFTL } \\
(\mathrm{n}=11)\end{array}$ & \multirow[t]{2}{*}{$\chi^{2}$} & \multirow[t]{2}{*}{$P$} \\
\hline & & $n(\%)$ & $n(\%)$ & & \\
\hline \multirow{2}{*}{$\begin{array}{c}\text { PAS } \\
(\mathrm{mmHg})\end{array}$} & Normal & $26(74,3)$ & $9(25,7)$ & \multirow{2}{*}{0,025} & \multirow{2}{*}{0,875} \\
\hline & Elevada & $5(71,4)$ & $2(28,6)$ & & \\
\hline \multirow{2}{*}{$\begin{array}{c}\text { PAD } \\
(\mathrm{mmHg})\end{array}$} & Normal & $29(76,3)$ & $9(23,7)$ & \multirow{2}{*}{1,296} & \multirow{2}{*}{0,255} \\
\hline & \begin{tabular}{|l|} 
Elevada \\
\end{tabular} & $2(50,0)$ & $2(50,0)$ & & \\
\hline \multirow{2}{*}{$\begin{array}{c}\text { RMSSD } \\
(\mathrm{ms})\end{array}$} & Baixa & $7(70,0)$ & $3(30,0)$ & \multirow{2}{*}{0,099} & \multirow{2}{*}{0,754} \\
\hline & Elevada & $24(75,0)$ & $8(25,0)$ & & \\
\hline \multirow{2}{*}{$\begin{array}{l}\mathrm{LF} \\
\text { (n.u.) }\end{array}$} & Baixo & $7(70,0)$ & $3(30,0)$ & \multirow{2}{*}{0,099} & \multirow{2}{*}{0,754} \\
\hline & Elevado & $24(75,0)$ & $8(25,0)$ & & \\
\hline \multirow{2}{*}{$\begin{array}{l}\mathrm{HF} \\
\text { (n.u.) }\end{array}$} & Baixo & $8(80,0)$ & $2(20,0)$ & \multirow{2}{*}{0,260} & \multirow{2}{*}{0,610} \\
\hline & Elevado & $23(71,9)$ & $9(28,1)$ & & \\
\hline \multirow{2}{*}{$\begin{array}{c}\mathrm{LF} / \mathrm{HF} \\
(\%)\end{array}$} & Baixo & $7(70,0)$ & $3(30,0)$ & \multirow{2}{*}{0,099} & \multirow{2}{*}{0,754} \\
\hline & Elevado & $24(75,0)$ & $8(25,0)$ & & \\
\hline
\end{tabular}

PAS $=$ pressão arterial sistólica; $\mathrm{PAD}=$ pressão arterial diastólica; RMSSD = raiz quadrada da média do quadrado das diferenças entre intervalos RR normais adjacentes; $L F=$ componente de baixa frequência; $\mathrm{HF}=$ componente de alta frequência; $\mathrm{LF} / \mathrm{HF}=$ razão entre componente de baixa frequência e componente de alta frequência.

Fonte: Dados da pesquisa.

O presente estudo se caracteriza como um ensaio de 
delineamento transversal que envolveu crianças e adolescentes de ambos os sexos, no qual se buscou analisar uma possível associação entre os diferentes domínios da PHAF com a PA e VFC.

A prática habitual de atividades físicas está diretamente relacionada a um maior nível de aptidão física. Dessa maneira a atividade física demonstra ser uma grande ferramenta para a promoção da saúde física. Sabe-se que indivíduos com maior prática de atividade física estão menos propensos a apresentar indicadores desfavoráveis de risco cardiovascular e doenças crônico-degenerativas (GUTIN et al., 2005).

No presente estudo, a amostra apresentou maior porcentual de indivíduos com baixa prática habitual de atividade física. A mesma foi avaliada em diferentes domínios (tempo livre, escola e lazer). Constata-se ainda que indivíduos inativos apresentam maiores valores de PA em repouso, em relação à seus pares fisicamente ativos (GUEDES; GUEDES; et al., 2006). Dado ao contexto emergem diversos estudos em diferentes linhas de pesquisa visando analisar e/ou estabelecer uma associação entre variáveis cardiovasculares e prática habitual de atividade física.

Crianças e adolescentes menos ativos podem apresentar maior probabilidade de PA elevada ( $80 \%$ a $90 \%$ ) quando comparados aos mais ativos (GUEDES; GUEDES; et al., 2006). É verificado ainda que o mesmo tipo de população quando fisicamente ativa apresenta menores valores de PA diastólica e PA sistólica (QUADROS et al., 2016). No presente estudo pode ser constatado que em diferentes domínios da PHAF a maior parte dos indivíduos que possuíam valores de PA elevada apresentou baixa PHAF.

O sistema nervoso autônomo tem sido implicado na hipertensão com atividade elevada do sistema nervoso simpático e diminuição da atividade do sistema nervoso parassimpático associada à etiologia, curso, progressão e consequências da hipertensão (BROOK; JULIUS, 2000). Nesse sentido, o sistema nervoso autônomo desempenha um importante papel no controle da pressão arterial e da frequência cardíaca. A avaliação da VFC tem se mostrado eficiente para analisar o comportamento sistema nervoso autônomo em diferentes condições fisiológicas e patológicas (GRANDEMANGE et al., 2019).

Sabe-se que a análise da ação simpática e parassimpática através das variações nos intervalos R-R é um mecanismo eficiente para analisar o controle autonômico cardíaco batimento a batimento (AKSELROD et al., 1987; SAUL et al., 1990). Nota-se nesse estudo que nos domínios PHAFE e PHAFL os indivíduos com alta PHAF apresentaram maiores valores de LF (indicador de variabilidade global) e LF/HF (indicador de balanço autonômico) e menores valores de HF (indicador parassimpático). Os mesmos valores foram observados na PHAF geral. Quanto ao domínio tempo livre os indivíduos com alta PHAF apresentaram menores valores de LF e LF/HF e maiores valores de HF.
Indivíduos que apresentam maior VFC possuem atividade do tônus parassimpático aumentada e redução do tônus simpático. Dessa maneira a alta VFC tende a proporcionar melhor capacidade de adaptação do sistema cardiovascular às condições de stress fisiológico (NAKAMURA et al., 2005). Observa-se no presente estudo que os valores de PHAFTL sugerem que os indivíduos mais ativos no referido domínio possuem maior proteção cardíaca, revelada pelos valores de HF.

Apesar de todo cuidado metodológico, alguns aspectos devem ser considerados. O delineamento transversal da presente investigação não permite estabelecer relação de causa-efeito no que tange aos impactos da prática habitual de atividade física na PA, bem como no comportamento autonômico (VFC). Além disso, diferenças maturacionais podem também em algum grau afetar as respostas analisadas. Por fim, o tamanho da amostra pode ter limitado a identificação de associações mais pronunciadas. Assim, embora não se tenha obtido diferenças estatisticamente significativas, é provável que com um maior número de sujeitos o poder estatístico se eleve, possibilitando a identificação de resultados de associação estatisticamente significativos.

Quanto aos pontos fortes, vale destacar que não é de nosso conhecimento estudo similar que buscou analisar a relação entre os diferentes domínios da prática habitual de atividades físicas com a PA e VFC em escolares da rede pública de ensino do Brasil. Adicionalmente, foi empregado delineamento do tipo categórico, afim de fortalecer as análises, uma vez que a categorização baseada em pontos de corte previamente definidos reduzem as limitações quanto aos aspectos maturacionais da presente amostra.

\section{Conclusão}

A prática geral de atividades físicas habituais, bem como seus diferentes domínios, sejam eles no lazer, escola e tempo livre, não se associaram à valores aumentados de pressão arterial, bem como com os indicadores do comportamento autonômico de crianças e adolescentes.

\section{Referências}

AKSELROD, S. et al. Hemodynamic regulation in SHR: investigation by spectral analysis. Am J. Physiol., v.253, n.1 Pt 2, p.H176-183, 1987. doi:10.1152/ajpheart.1987.253.1.H176.

BAECKE, J. A.; BUREMA, J.; FRIJTERS, J. E. A short questionnaire for the measurement of habitual physical activity in epidemiological studies. Am J Clin Nutr. v.36, n.5, p.936-942, 1982. doi:10.1093/ajen/36.5.936

BROOK, R. D.; JULIUS, S. Autonomic imbalance, hypertension, and cardiovascular risk. Am J Hypertens. v.13, n.6, p.112S-122S, 2000. doi:10.1016/s0895-7061(00)00228-4

CHRISTOFARO, D. G. et al. Evaluation of the Omron MX3 Plus monitor for blood pressure measurement in adolescents. Eur $J$ Pediatr. v.168, n.11, p.1349-1354, 2009. doi:10.1007/s00431009-0936-X

FLYNN, J. T. et al. Clinical Practice Guideline for Screening and 
Management of High Blood Pressure in Children and Adolescents. Pediatrics. v.140, n.3, 2017. doi:10.1542/peds.2017-1904

GOIT, R. K.; ANSARI, A. H. Reduced parasympathetic tone in newly diagnosed essential hypertension. Indian Heart J. v.68, n.2, p.153-157, 2016. doi:10.1016/j.ihj.2015.08.003

GORDON, C. C.; CHUMLEA, W. C.; ROCHE, A. F. Stature, recumbent length, and weight. Champaign: Human Kinetics Books, 1988. 3-8 p. (Anthropometric standardization reference manual).

GRANDEMANGE, M. et al. Blood Pressure, Heart Rate Variability, and Adiposity in Caribbean Pre-pubertal Children. Front Pediatr. v.7, p.269, 2019. doi:10.3389/fped.2019.00269

GUEDES, D. P. et al. Cardiovascular risk factors in adolescents: biological and behavioral indicators. Arq Bras Cardiol. v.86, n.6, p.439-450, 2006.

GUEDES, D. P. et al. Reprodutibilidade e validade do questionário Baecke para avaliação da atividade física habitual em adolescentes. Rev Port Cien Desp. v.6, n.3, p.265-274, 2006.

GUTIN, B. et al. Relations of moderate and vigorous physical activity to fitness and fatness in adolescents. Am J Clin Nutr. v.81, n.4, p.746-750, 2005. doi:10.1093/ajen/81.4.746

Heart rate variability: standards of measurement, physiological interpretation and clinical use. Task Force of the European Society of Cardiology and the North American Society of Pacing and Electrophysiology. Circulation. v.93, n.5, p.1043-1065, 1996.

HEFFERNAN, K. S. et al. Heart rate recovery and heart rate complexity following resistance exercise training and detraining in young men. Am J Physiol Heart Circ Physiol. v.293, n.5, p.H3180-3186, 2007. doi:10.1152/ajpheart.00648.2007

JOYCE, D.; BARRETT, M. State of the science: heart rate variability in health and disease. BMJ Support Palliat Care. v.9, n.3, p.274-276, 2019. doi:10.1136/bmjspcare-2018-001588

KIVINIEMI, A. M. et al. Cardiac autonomic function and high-intensity interval training in middle-age men. Med Sci Sports Exerc. v.46, n.10, p.1960-1967, 2014. doi:10.1249/ MSS.0000000000000307

KUNG, H. C.; XU, J. Hypertension-related Mortality in the United States, 2000-2013. NCHS Data Brief. n.193, p.1-8, 2015.

LEES, T. et al. Heart Rate Variability as a Biomarker for Predicting Stroke, Post-stroke Complications and Functionality. Biomark Insights. v.13, p.1177271918786931, 2018.

\section{doi:10.1177/1177271918786931}

LEHRER, P. et al. Heart Rate Variability Biofeedback Improves Emotional and Physical Health and Performance: A Systematic Review and Meta Analysis. Appl Psychophysiol Biofeedback. 2020. doi:10.1007/s10484-020-09466-Z

NAKAMURA, F. Y. et al. Alteração do limiar de variabilidade da frequência cardíaca após treinamento aeróbio de curto prazo. Motriz: Rev Educ Fis., v.11, n.1, p.1-9, 2005.

PICKERING, T. G. et al. Recommendations for blood pressure measurement in humans and experimental animals: part 1: blood pressure measurement in humans: a statement for professionals from the Subcommittee of Professional and Public Education of the American Heart Association Council on High Blood Pressure Research. Circulation. v.111, n.5, p.697-716, 2005. doi:10.1161/01.CIR.0000154900.76284.F6

PLEWS, D. J. et al. Training adaptation and heart rate variability in elite endurance athletes: opening the door to effective monitoring. Sports Med. v.43, n.9, p.773-781, 2013. doi:10.1007/ s40279-013-0071-8

QUADROS, T. M. et al. [Epidemiological survey in schoolchildren: determinants and prevalence of cardiovascular risk factors]. Cad Saude Publica. v.32, n.2, p.e00181514, 2016. doi:10.1590/0102-311X00181514

QUINTANA, D. S.; HEATHERS, J. A.; KEMP, A. H. On the validity of using the Polar RS800 heart rate monitor for heart rate variability research. Eur J Appl Physiol. v.112, n.12, p.41794180, 2012. doi:10.1007/s00421-012-2453-2

SAUL, J. P. et al. Heart rate and muscle sympathetic nerve variability during reflex changes of autonomic activity. Am $J$ Physiol. v.258, n.3 Pt 2, p.H713-721, 1990. doi:10.1152/ ajpheart.1990.258.3.H713

SEN, J.; MCGILL, D. Fractal analysis of heart rate variability as a predictor of mortality: A systematic review and meta-analysis. Chaos. v.28, n.7, p.072101, 2018. doi:10.1063/1.5038818

SOARES-MIRANDA, L. et al. Physical activity and heart rate variability in older adults: the Cardiovascular Health Study. Circulation. v.129, n.21, p.2100-2110, 2014. doi:10.1161/ CIRCULATIONAHA.113.005361

STEIN, P. K. et al. Effect of exercise training on heart rate variability in healthy older adults. Am Heart J. v.138, n.3 Pt 1, p.567-576, 1999. doi:10.1016/s0002-8703(99)70162-6 